\title{
Parameter selection in dynamic contrast-enhanced magnetic resonance tomography
}

\author{
Niinimäki, K and Hanhela, M and Kolehmainen, V
}

\begin{abstract}
In this work we consider the image reconstruction problem of sparsely sampled dynamic contrast-enhanced (DCE) magnetic resonance imaging (MRI). DCE-MRI is a technique for acquiring a series of MR images before, during and after intravenous contrast agent administration, and it is used to study microvascular structure and perfusion. To overcome the ill-posedness of the related spatiotemporal inverse problem, we use regularization. In regularization one of the main problems is how to determine the regularization parameter which controls the balance between data fitting term and regularization term. Most methods for selecting this parameter require the computation of a large number of estimates even in stationary problems. In dynamic imaging, the parameter selection is even more time consuming since separate regularization parameters are needed for the spatial and temporal regularization functionals. In this work, we study the possibility of using the S-curve with DCE-MR data. We select the spatial regularization parameter using the S-curve, leaving the temporal regularization parameter as the only free parameter in the reconstruction problem. In this work, the temporal regularization parameter is selected manually by computing reconstructions with several values of the temporal regularization parameter.
\end{abstract}

\section{Introduction}

Dynamic contrast-enhanced MRI (DCE-MRI) is an imaging method which is used to study microvascular structure and tissue perfusion. The method has many applications including blood-brain-barrier assessment after acute ischemic stroke [21, 30] and treatment monitoring of breast cancer [20, 23] and glioma [24]. The operation principle of DCE-MRI is to inject a bolus of gadolinium based contrast agent into the blood stream, and acquire a time series of MRI data with a suitable $T_{1}$-weighting to obtain a time series of $2 \mathrm{D}$ (or 3D) images which exhibit contrast changes induced by concentration changes of the contrast agent in the tissues. 
The analysis of the contrast agent dynamics during imaging requires high resolution in space and in time; the high temporal resolution is necessary to measure when the contrast is passing through the artery and it is used for determining the subject specific Arterial Input function (AIF), and the high spatial resolution is necessary to adequately capture boundaries of perfused tissues. In many cases, sufficient time resolution can only be obtained by utilizing an imaging protocol during which only partial k-space sampling can be obtained for each image in the time series. However, acquiring less samples than required by the Nyquist criterion makes the image reconstruction problem ill-posed (and non-unique), causing artifacts and deterioration of the image quality when conventional reconstruction methods such as inverse Fourier transform or re-gridding are employed.

According to the theory of compressed sensing (CS) [6, 7, 8], images that have a sparse representation can be recovered from undersampled measurements of a linear transform, i.e. sampling rate below Nyquist rate, using appropriate nonlinear reconstruction algorithms and appropriate (random) sampling of the data space. The compressibility of MR images and the fact that MR scanner measures samples of a linear transform of the unknown image (the k-space samples can be mathematically considered as Fourier coefficients) suggest that the idea of CS is applicable to MR imaging, offering thus a potentially significant scan time reduction without sacrificing the image quality. Since the seminal work of Candès, Romberg and Tao in 2006, CS has been extensively applied to MRI. In 2007 CS was applied to MRI in [19] and in 2018 it received FDA approval for clinical use.

The undersampling of the k-space for speeding up the dynamic MRI data acquisition can in principle be done in many different ways. However, for many applications, the low frequency features that are present in the center of the k-space are of importance. In [33], for example, it was demonstrated that center weighted random sampling patterns were preferable to purely random sampling of the $\mathrm{k}$-space within the CS approach. Radial sampling has the advantage that the center of the $\mathrm{k}$-space is sampled densely, even when the sampling (i.e., number of radial spokes) is remarkably reduced.

In this paper, we consider image reconstruction problem of DCE-MRI with sparsely sampled golden angle radial data, where the angle of subsequent spokes is $\sim 111.25^{\circ}$. The number of measurement spokes used for reconstructing a single time frame is chosen to be a Fibonacci number, which was shown to be an optimal choice in [32]. This type of sparse sampling between the time frames results in each time frame having different spokes, and eventually the spokes cover the whole k-space.

We also combine the Golden Angle (GA) sampling with concentric squares sampling. This sampling strategy resembles the linogram method [10] developed for computed tomography imaging, but the angles of subsequent spokes were chosen according to the golden angle method as opposed to the angles being equidistant in $\tan \theta$ in linogram sampling. Unlike the conventional radial sampling pattern with spokes of equal length, the concentric squares sampling strategy also covers the corners of the k-space. The sampling pattern therefore also collects information of the 
high frequencies in the corners of the k-space, which leads to a reduction of artifacts originating from the lack of sampling in the corners.

To overcome the ill-posedness of our image reconstruction problem, we use a variational framework. Thus we solve the following optimization problem

$$
\hat{f}=\arg \min D(f, m)+\alpha S(f)+\beta T(f),
$$

where $f=\left\{f_{1}, f_{2}, \ldots, f_{N_{t}}\right\}$ denotes the image sequence, $m=\left\{m_{1}, m_{2}, \ldots, m_{N_{t}}\right\}$ the data sequence, $N_{t}$ the number of time frames, $D$ the data-fitting term, $S$ the spatial regularization, $T$ the temporal regularization and $\alpha$ and $\beta$ are the spatial and temporal regularization parameters, respectively. The selection of the regularization parameters is crucial in terms of resulting image quality. There exists several proposed parameter selection methods, but most of them require the computation of a large number of reconstructions with varying parameters. Having to fix two regularization parameters makes the selection even more time consuming.

In this work, we propose to use the S-curve method [13, 15, 17, 22] for automatic selection of the spatial regularization parameter $\alpha$. The idea in the S-curve method is to select the regularization parameter so that the reconstruction has a priori defined level of sparsity in the chosen transformation domain. In DCE-MRI, a reliable a priori estimate for the sparsity level can be extracted from an anatomical MRI image which is based on full-sampling of the k-space and is always taken as part of the MRI measurement protocol but is usually used only for visualization purposes. For the selection of the spatial regularization parameter $\alpha$, we employ one time frame of the GA data from the baseline measurement before the contrast agent administration. After fixing the spatial regularization parameter, we compute dynamic reconstructions with several values of parameter $\beta$ and select a suitable temporal regularization parameter manually. Furthermore we study the performance of three different temporal regularization functionals, namely temporal smoothness, temporal total variation and total generalized variation.

The proposed method is evaluated using simulated GA DCE-MRI data from a rat brain phantom. The results are compared to re-gridding approach, which is the most widely used non-iterative algorithm for reconstructing images from non-Cartesian MRI data. Our re-gridding method was developed in IR4M UMR8081, CNRS, Université Paris-Sud using Matlab ${ }^{\circledR}$. This re-gridding approach does not need additional density correction and it was first used in [27], see also [12].

\section{Image reconstruction in radial DCE-MRI}

\subsection{Forward problem}

The forward problem in 2D MRI can be modelled for most measurement protocols by the Fourier transform 


$$
m\left(k_{x}, k_{y}\right)=\int_{\Omega} f(x, y) e^{-i 2 \pi\left(k_{x} x+k_{y} y\right)} \mathrm{d} x \mathrm{~d} y,
$$

where $\Omega$ is the image domain $f(x, y)$ is the unknown image, $m\left(k_{x}, k_{y}\right)$ is the measured data, and $k_{x}, k_{y}$ denotes the k-space trajectories. In the discrete framework, the Fourier transform is typically approximated with the multidimensional FFT when using cartesian k-space trajectories and with the non-uniform FFT when using noncartesian k-space trajectories.

In this work, we consider non-uniform k-space trajectories and approximate the Fourier transform by the non-uniform fast Fourier transform (nuFFT) operator [11]. We discretize our functions as follows; temporal direction is divided into a sequence of $N_{t}$ (vectorized) images $f=\left\{f_{1}, f_{2}, \ldots, f_{N_{t}}\right\}$ and data vectors $m=\left\{m_{1}, m_{2}, \ldots, m_{N_{t}}\right\}$, where each $f_{t} \in \mathbb{C}^{N_{p}}$ and $m_{t} \in \mathbb{C}^{M}$, respectively. The number of data per frame $M$ is equal to the number of GA spokes per frame times the number of samples per spoke. The number of image pixels is $N_{p}=N \times N$. Thus, using nuFFT we re-write (2) in a discretized form at time $t$ as

$$
m_{t}=A_{t} f_{t}+\varepsilon_{t}, \quad t=1, \ldots, N_{t},
$$

$A_{t}=P_{t} \mathscr{F} S_{t}$, where $P_{t}$ is an interpolation matrix between Cartesian k-space and noncartesian k-space, $\mathscr{F}$ is the $2 \mathrm{D}$ FFT operation and $S_{t}$ is a scaling matrix.

\subsection{Inverse problem of dynamic image reconstruction}

The dynamic inverse problem related to the equation (3) is: given measurement time series $m=\left\{m_{1}, m_{2}, \ldots, m_{N_{t}}\right\}$ and the associated k-space trajectories, solve the unknown images $f=\left\{f_{1}, f_{2}, \ldots, f_{N_{t}}\right\}$. n. To recover $f$ from $m$, we define the inverse problem as the optimization problem

$$
\hat{f}=\arg \min \left\{\sum_{t=1}^{N_{t}}\left\|A_{t} f_{t}-m_{t}\right\|_{2}^{2}+\alpha S(f)+\beta T(f)\right\},
$$

where $S(f)$ denotes the spatial regularization functional, $\alpha$ the spatial regularization parameter, $\beta$ the temporal regularization parameter and $T(f)$ the temporal regularization functional.

In this work, we study the applicability of S-curve method for selecting the spatial regularization parameter $\alpha$. Once $\alpha$ has been fixed, the temporal regularization parameter is then selected manually by computing estimates with different values of $\beta$. Our minimization problem is based on $L_{2}$-data fidelity term for the measurement model and we use spatial total variation regularization for promoting sparsity of the gradients of each image [26]. Furthermore we study the performance of three different temporal regularization functionals for promoting temporal regularity of the image series. Our spatio-temporal image reconstruction problem thus writes 


$$
\hat{f}=\arg \min \left\{\sum_{t=1}^{N_{t}}\left(\left\|A_{t} f_{t}-m_{t}\right\|_{2}^{2}+\alpha\left\|\nabla f_{t}\right\|_{2,1}\right)+\beta T(f)\right\},
$$

where the isotropic 2D spatial total variation norm for complex valued image $f_{t}$ is defined by

$$
\|\cdot\|_{2,1}=\sum_{k=1}^{N} \sqrt{\left(\operatorname{Re}\left(D_{x} f_{t, k}\right)\right)^{2}+\left(\operatorname{Re}\left(D_{y} f_{t, k}\right)\right)^{2}+\left(\operatorname{Im}\left(D_{x} f_{t, k}\right)\right)^{2}+\left(\operatorname{Im}\left(D_{y} f_{t, k}\right)\right)^{2}},
$$

where $R e$ and $I m$ denoting the real and imaginary parts of $f_{t}$ respectively and $D_{x}$ and $D_{y}$ the discrete forward first differences in horizontal and vertical directions, respectively.

\subsubsection{Temporal regularization 1: Temporal smoothness (TS)}

The temporal smoothness regularization (hereafter referred as TS) is defined as the $L_{2}$ norm of forward first differences in time:

$$
T(f)=\sum_{t=1}^{N_{t}-1}\left\|f_{t+1}-f_{t}\right\|_{2}^{2} .
$$

This model promotes smooth slowly changing signals, and it has been used in [3] for radial DCE myocardial perfusion imaging. TS regularization was compared with temporal TV regularization in the same application in [1].

\subsubsection{Temporal regularization 2 : Temporal total variation (TV)}

Temporal total variation (hereafter referred as TV) is defined by the $L_{1}$ norm of the forward first differences in time:

$$
T(f)=\sum_{t=1}^{N_{t}-1}\left\|f_{t+1}-f_{t}\right\|_{1}
$$

The temporal total variation model promotes sparsity of the time derivative of the pixel signals, being a highly feasible regularization model for reconstruction of piece-wise regular signals which may exhibit large jumps. The smoothed form of temporal total variation was used in [2] for multislice myocardial perfusion imaging.

\subsubsection{Temporal regularization 3: Total generalized variation (TGV)}

The total generalized variation model [4] is a total variation model that is generalized to higher order differences. Here we use the second-order total generalized 
variation, which in the discrete 1-dimensional form is of the form

$$
T(f)=\sum_{t=1}^{N_{t}-1} \min _{v}\left\|f_{t+1}-f_{t}-v_{t}\right\|_{1}+\gamma\left\|v_{t+1}-v_{t}\right\|_{1},
$$

where $v$ is an auxiliary vector and $\gamma$ is a parameter, which balances the first and second order terms, and is set to $\sqrt{2}$ here.

This functional balances between minimizing the first-order and second-order differences of the signal. The difference with TV regularization is most clear in smooth regions where piecewise linear solutions are favored over the piecewise constant solutions of TV. From hereafter this temporal regularization is referred as TGV.

TGV was first used in MRI as a spatial prior in [16], and it has also been used in DCE-MRI as a temporal prior in [31], where different temporal priors were compared in cartesian MRI of the breast.

\subsection{Regularization parameter selection}

\subsubsection{Spatial regularization parameter selection}

The spatial regularization parameter is selected using the S-curve method, originally proposed in [13, 15, 17, 22], but here modified for TV regularization.

Assume that we have an a priori estimate $\hat{S}$ for the total variation norm of the unknown function. In practice we can use an anatomical image of the same slice in order to obtain a reliable estimate for $\hat{S}$. Such an anatomical image is practically always acquired as part of the DCE MRI acquisition experiment but usually only used for visualization purposes. However, if such an anatomical image was not acquired, we could, in case of GA acquisition, estimate the expected sparsity level from a conventional reconstruction of a long sequence of baseline data taken before the contrast agent injection. Or the anatomical image could be estimated from the entire data-acquisition similarly as the composite image in [5].

Now, given the estimate $\hat{S}$ we select the regularization parameter $\alpha$ using the S-curve method as follows

1) Take a sequence of regularization parameters $\alpha$ ranging on the interval $[0, \infty]$ such that

$$
0<\alpha^{(1)}<\alpha^{(2)}<\cdots<\alpha^{(L)}<\infty .
$$

2) Compute the corresponding estimates $\hat{f}_{1}\left(\alpha^{(1)}\right), \ldots, \hat{f}_{1}\left(\alpha^{(L)}\right)$.

With DCE-MRI data, reconstructions $\hat{f}_{1}\left(\alpha^{(\ell)}\right)$ are computed as follows; we take the data that correspond to the first time frame, i.e. $m_{1}$ which has number of elements equal to the number of GA spokes per frame times the number of points per spoke. We reconstruct $f_{1}$ for given value $\alpha^{(\ell)}$ by

$$
\hat{f}_{1}\left(\alpha^{(\ell)}\right)=\arg \min _{f_{1}}\left\{\left\|A_{1} f_{1}-m_{1}\right\|_{2}^{2}+\alpha^{(\ell)}\left\|\nabla f_{1}\right\|_{2,1}\right\} .
$$


Here it is important that $\alpha^{(1)}$ is taken to be so small that the problem is under regularized and the corresponding reconstruction $\hat{f}_{1}\left(\alpha^{(1)}\right)$ results to a very noisy image with a big TV-norm value and $\alpha^{(L)}$ is taken so large that the problem is over regularized and TV norm of reconstruction $\hat{f}_{1}\left(\alpha^{(L)}\right)$ is very close to zero.

3) Compute the TV-norms of the recovered estimates $\hat{f}_{1}\left(\alpha^{(\ell)}\right), \quad \ell=1, \ldots, L$.

4) Fit a smooth interpolation curve to the data $\left\{\alpha^{(\ell)}, S\left(\alpha^{(\ell)}\right), \quad \ell=1, \ldots, L\right\}$ and use the interpolated sparsity curve to find the value of $\alpha$ for which $S(\alpha)=\hat{S}$. For the interpolation we use Matlab's ${ }^{\circledR}$ interp function and we interpolate our original S-curve to a more dense discretization of the regularization parameter $\alpha$.

\subsection{Temporal regularization parameter selection}

Once the spatial regularization parameter $\alpha$ has been fixed using the S-curve, the temporal regularization parameter $\beta$ can be tuned by computing estimates with different values of $\beta$ and selecting a suitable value manually, for example, by visual assessment of the results.

In this work, we compute the results with three different temporal regularization models using simulated measurement data. Since we consider a simulated test case where a ground truth is available, we select an optimal value of $\beta$ for each temporal regularization model by selecting the value of $\beta$ which produces the reconstruction with the smallest root mean square error (RMSE). The RMSE values were calculated separately for three regions; tumor, vascular region and the rest of the image domain. The RMSEs of different ROIs were then used to define a joint RMSE as

$$
R M S E_{\text {joint }}=\sqrt{R M S E_{\Omega_{\text {roi } 1}}^{2}+R M S E_{\Omega_{\text {roi }}}^{2}+R M S E_{\Omega_{\text {roij }}}^{2}},
$$

where $\Omega_{\text {roil }}$ corresponds to pixels in the vascular region, $\Omega_{\text {roi } 2}$ correspond to pixels in the tumour region and $\Omega_{\text {roi3 }}$ corresponds to pixels in rest of the image domain. The RMSE was calculated this way to weigh the small tumour and vascular regions appropriately. In estimating the pharmacokinetic parameters of tissues, obtaining an accurate arterial input function (AIF) is required [28]. The AIF can be obtained via population averaging, however, usage of patient specific AIF produces more accurate estimates of the kinetic parameters [25]. The AIF is preferably extracted from an artery feeding the tissues of interest, but it can also be estimated from a venous sinus or vein when an artery is not visible [18]. Here the AIF is estimated from the superior sagittal sinus.

\section{Materials and methods}

A simulated test case modelling DCE-MRI measurements of a glioma in rat brain was created. The rat brain phantom was based on the rat brain atlas in [29], and 
scaled to a size of $128 \times 128$. The rat brain image was divided into three subdomains of different signal behaviour: vascular region (labelled ' 1 ' and highlighted with red in figure 11), tumour region (labelled '2' and highlighted with blue in figure 11) and the rest of the brain tissue. The vascular signal region corresponds to the location of the superior sagittal sinus.

A time series of 2800 ground truth images was simulated by multiplying the signal of each pixel with the template of the corresponding region and adding that to the baseline value of the pixels. The tumour signal templates were based on an experimental DCE-MRI measurement described in [14], where the three different ROIs were identified. Figure 1 shows the signal templates for each of the different tissue regions.

One spoke of GA k-space data was simulated for each of the simulated images, leading to a dynamic experiment with 2800 spokes of $\mathrm{k}$-space data. The time scale of the simulation was set to be similar to the in vivo measurements in [14] where the measurement time between consecutive GA spokes was $38.5 \mathrm{~ms}$. Gaussian complex noise at $5 \%$ of the mean of the absolute values of the signal was added to the simulated $\mathrm{k}$-space signal. The simulated test case was carried out using a $\mathrm{k}$-space trajectory which combines the golden angle and the concentric squares sampling strategies.

In [14] it was found that reconstruction of the form (5) performed optimally with segment length 1 of 34 for a similar data set, thus we selected 34 as the segment length for our reconstructions leading to a temporal resolution of $\sim 1.3 \mathrm{~s}$.

All the regularized reconstructions in this work were computed using the ChambollePock primal-dual algorithm [9]. In the NUFFT implementation of the forward model, the measurements were interpolated into a twice oversampled cartesian grid with min-max Kaiser-Bessel interpolation with a neighbourhood size of 4 [11]. The regridding reconstructions were computed using a Matlab code developped at Imagerie par résonance magnétique médicale et multi-modalités (IR4M) UMR8081, Université Paris-Sud, France.

We remark that when computing the RMS error [10], the reconstructed time signals of each pixel were linearly interpolated in the temporal direction to match the temporal resolution of the ground truth phantom.

\section{Results}

The selection of $\alpha$ was carried out using the first 34 spokes (i.e. the first frame $m_{1}$ ) of the DCE-MRI data and then the selected $\alpha$ was used for all the spatio-temporal reconstructions with different temporal regularizations. The rat brain phantom of section 3 was used to compute the a priori level of sparsity, i.e. in our case we computed the TV norm of the first time frame of the dynamic phantom. This resulted in a sparsity level of $\hat{S}=0.0259$. The spatial regularization term $\alpha$ was selected

\footnotetext{
${ }^{1}$ Segment length equals the number of radial spokes per image. The number of elements $M$ in the data vector $m_{t}$ is segment length times number of samples per spoke.
} 

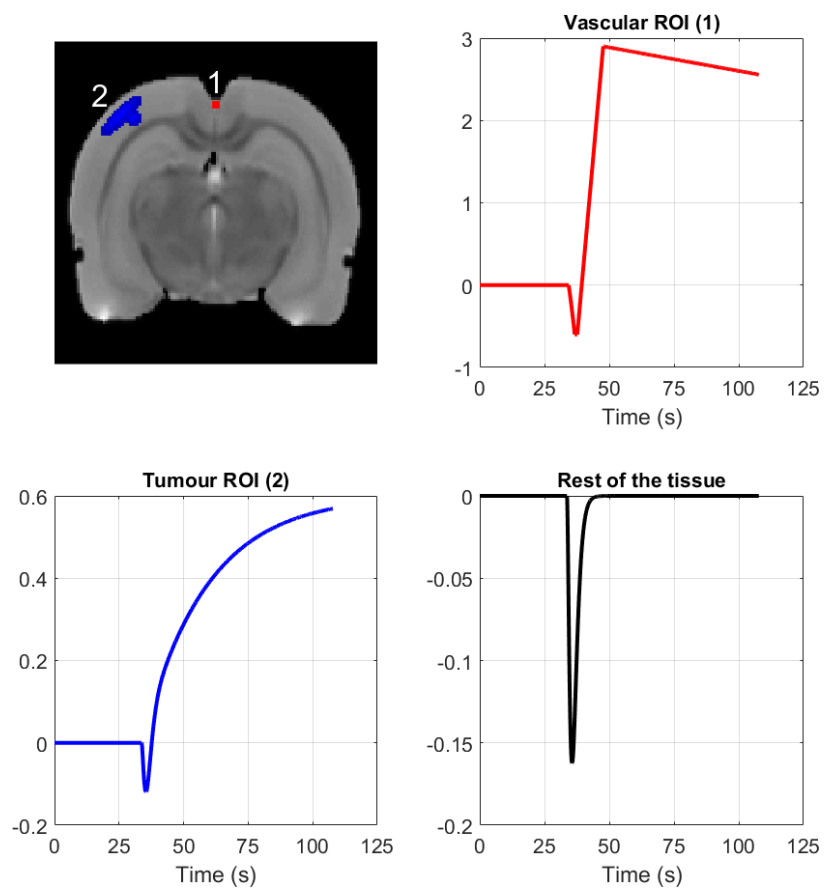

Fig. 1 Template signals of different ROIs. Top left: The simulated image with vascular ROI labelled ' 1 ' and marked with red and tumour ROI labelled ' 2 ' and marked with blue. Top right: The template signal of the vascular ROI. Bottom left: The template signal of the tumour ROI. Bottom right: The template signal of tissue outside the two ROIs. The vertical axis in the three template signals is the multiplier for the signal added to the base signal.

using the S-curve as described in section 2.3.1 and resulted in spatial regularization parameter value of $\alpha=7.3 e-4$. The TV norms of the reconstructions for the Scurve were computed with 15 values of alpha ranging on interval $\left[10^{-7}, 10^{3}\right]$. These 15 values of TV norm were then interpolated using Matlab's ${ }^{\circledR}$ interp function to 405 values. The resulting S-curve for the determination of $\alpha$ is presented in figure 2 .

In many practical applications, the a priori information, which we use to estimate the value of $\hat{S}$, may come from a different modality or from acquisition with different pulse sequence than the one used in the dynamic measurement. Therefore, in order to compute meaningful estimate of $\hat{S}$ for the TV-regularized case, the reference image has to be scaled such that it is compatible with the measured dynamic data. This normalization of the reference image can be obtained by

$$
f_{\mathrm{ref}}=\frac{\left\|m_{t}\right\|}{\left\|A_{t} f_{\mathrm{ref}}\right\|} f_{\mathrm{ref}},
$$

where $f_{\text {ref }}$ denotes the reference image, $m_{t}$ the frame of dynamic data that is used in the $\mathrm{S}$-curve and $A_{t}$ the respective forward model. 


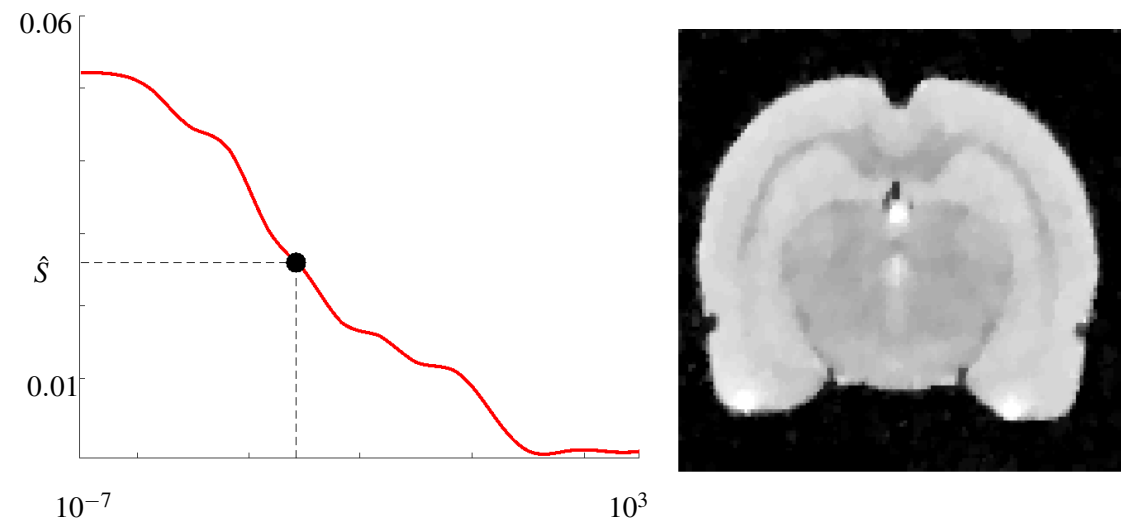

Fig. 2 S-curve for selecting spatial regularization parameter for the simulated data case. Left: Plot of the interpolation curve used to determine the value of $\alpha$ such that $S(\alpha)=\hat{S}=0.0259$. Right: Reconstruction (resolution $128 \times 128)$ of the first time frame $(t=1)$ using the selected value of $\alpha=7.3 e-4$.

The temporal regularization parameter $\beta$ was selected by computing reconstructions (5) with different values of $\beta$ and then selecting the values which had the smallest joint RMSE error, see $(10)$.

This selection resulted in smallest joint RMSE of 0.085 for TS model which corresponded to $\beta=0.01$, smallest joint RMSE of 0.058 for the TV model corresponding to $\beta=0.0017$ and smallest joint RMSE of 0.063 , corresponding to $\beta=0.0022$ for the TGV model. As a reference method we selected the regularization parameters $\alpha$ and $\beta$ using L-surface method for the case where we use spatial total variation and temporal total variation as our regularization model. The resulted L-surface is presented in figure 3 Application of L-surface on our data resulted parameters $\alpha=3.1623 e-4$ and $\beta=3.1623 e-6$.

Figure 4 shows slices of all reconstructions before, during and after contrast agent administration. Figure 5 shows the reconstructed images with different methods for one time frame. The top row of figure 5 shows the phantom with a red square denoting a domain that is presented as a closeup in figure 6 for all of the reconstructions.

As can be seen from figures 4- 6, the classical regridding method fails on such high time resolution data as employed here, and thus we leave out the regridded reconstruction from the figures of the temporal evolution of the ROI signals. However the L-surface method seems to work nicely on our data, so we include it in the temporal evolution studies. The temporal evolutions in the vascular domain and in the tumor region are averages of $\Omega_{r o i 1}$ and $\Omega_{r o i 2}$, respectively. 


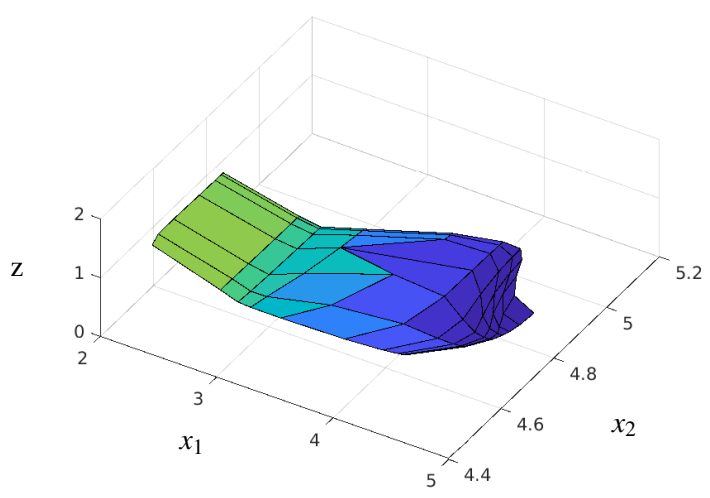

Fig. 3 L-surface for selecting the spatial regularization parameter and the temporal regularization parameter simultaneously, resulting in $\alpha=3.1623 e-4$ and $\beta=3.1623 e-6$. The axes in the image are: $x_{1}=\log \left(\left\|f_{t+1}-f_{t}\right\|\right), x_{2}=\log \left(\left\|\nabla f_{t}\right\|_{2,1}\right)$ and $z=\log \left(\left\|A_{t} f_{t}-m_{t}\right\|_{2}\right)$

Figure 7 shows the time signals of the reconstructions in the vascular region $\left(\Omega_{\text {roil }}\right)$ with the different temporal regularization models. Corresponding signals of the reconstructions in the tumor region (i.e. in $\Omega_{\text {roi } 2}$ ) are shown in figure 8 , The tumor region is accurately reconstructed with all the methods, with only small differences between the methods, L-surface method being the noisiest. In the vascular region, the methods have some differences with TGV having the best reconstruction quality and TS having the worst reconstruction quality. The TS method shows smoothing at both the maximum and minimum signal levels whereas TGV reconstructs the fast signal change of the vascular region most reliably. L-surface method is again more noisy than the other reconstructions in the vascular region and its performance of reconstructing the vascular signal falls between TS and TV most likely due to the temporal regularization parameter being too small whereas the spatial regularization parameter is on the same order of magnitude as the parameter obtained with S-curve.

\section{Conclusions}

Variational regularization based solutions for dynamic MRI problems usually include two regularization parameters, one for the spatial and one for the temporal regularization, that the user has to select. Typically, the selection of both of the parameters is carried out manually based on visual assessment of the reconstructed im- 
ages. In this work we proposed to use the S-curve method for the automatic selection of the spatial regularization parameter, leaving the time regularization parameter the only free parameter. The S-curve method selects the regularization parameter based on the expected sparsity of unknown images in domain of the regularization functional. Furthermore, the method requires computation of the reconstructions with relatively few values of the parameter, making it computationally efficient. The approach was demonstrated to lead to a feasible choice of the spatial regularization parameter in a simulated DCE-MRI experiment of rat brain. The reconstructions were also computed with three different temporal regularization models with the same fixed spatial regularization parameter, demonstrating the robustness of the approach with different time regularization models.

While we proposed automatic selection of the spatial regularization parameter, the temporal regularization parameter was still selected manually. In this work, we selected the temporal regularization parameter by computing RMS errors with respect to a ground truth. If on the other hand the ground truth is unknown, the temporal regularization parameter could be selected based on visual assessment of reconstructed images, leaving $\beta$ to be the only free parameter. In the future work, we aim to study methods for automatic selection of the time regularization parameter as well. One possibility could be to extend the S-curve to select a parameter which leads to an expected sparsity level in the domain of the temporal regularization. A feasible estimate for the expected level of sparsity in the time direction could potentially be extracted from the changes in the dynamic measurement data.

\section{Acknowledgments}

This work was supported by Jane and Aatos Erkko foundation and the Academy of Finland, Centre of Excellence in Inverse Modelling and imaging (project 312343).

\section{References}

1. Ganesh Adluru and Edward V. R. DiBella. A comparison of L1 and L2 norms as temporal constraints for reconstruction of undersampled dynamic contrast enhanced cardiac scans with respiratory motion. In Proc. International Society for Magnetic Resonance in Medicine, volume 16, page 340, 2008.

2. Ganesh Adluru, Chris McGann, Peter Speier, Eugene G. Kholmovski, Akram Shaaban, and Edward V. R. Dibella. Acquisition and reconstruction of undersampled radial data for myocardial perfusion magnetic resonance imaging. Journal of Magnetic Resonance Imaging, 29(2):466-473, 2009.

3. Ganesh Adluru, Ross T. Whitaker, and Edward V. R. DiBella. Spatio-temporal constrained reconstruction of sparse dynamic contrast enhanced radial MRI data. In Proc. IEEE International Symposium on Biomedical Imaging, pages 109-112, 2007. 
4. Kristian Bredies, Karl Kunisch, and Thomas Pock. Total generalized variation. SIAM Journal on Imaging Sciences, 3(3):492-526, 2010.

5. Mistretta C.A., Wieben O., Velikina J., Block W., Perry J., Wu Y., Johnson K., and Wu Y. Highly constrained backprojection for time-resolved mri. Magn Reson Med, 55(1):30-40, 2006.

6. E. Candès, K. J. Romberg, and T. Tao. Stable signal recovery from incomplete and inaccurate measurements. Communications on Pure and Applied Mathematics, 59(8):1207-1223, August 2006.

7. E. J. Candès, J. Romberg, and T. Tao. Robust uncertainty principles: exact signal reconstruction from highly incomplete frequency information. IEEE Transactions on Information Theory, 52:489-509, 2006.

8. Emmanuel J. Candès, Justin K. Romberg, and Terence Tao. Stable signal recovery from incomplete and inaccurate measurements. Comm. Pure Appl. Math., 59(8):1207-1223, 2006.

9. Antonin Chambolle, Stacey E. Levine, and Bradley J. Lucier. An upwind finite-difference method for total variation-based image smoothing. SIAM J. Imaging Sci., 4(1):277-299, 2011.

10. Paul R. Edholm and Gabor T. Herman. Linograms in image reconstruction from projections. IEEE Transactions on Medical Imaging, 6(4):301-307, 1987.

11. J. A. Fessler and B. P. Sutton. Nonuniform fast fourier transforms using min-max interpolation. IEEE Transactions on Signal Processing, 51:560-574, 2003.

12. Guillot G. and Giovannelli. Iddn.fr.001.080011.000.s.p.2019.000.31230. International Identifier of Digital Works, 2 2019. An optional note.

13. K. Hämäläinen, A. Kallonen, V. Kolehmainen, M. Lassas, K. Niinimäki, and S. Siltanen. Sparse tomography. SIAM Journal on Scientific Computing, 35(3):B644-B665, 2013.

14. M. Hanhela, M. Kettunen, O. Gröhn, M. Vauhkonen, and V. Kolehmainen. Temporal huber regularization for dce-mr. submitted to Journal of Mathematical Imaging and Vision manuscript, 2019.

15. Niinimäki K. Computational Optimization Methods for Large-scale Inverse Problems. PhD thesis, University of Eastern Finland, 2013.

16. Florian Knoll, Kristian Bredies, Thomas Pock, and Rudolf Stollberger. Second order total generalized variation (TGV) for MRI. Magnetic Resonance in Medicine, 65(2):480-491, 2011.

17. V. Kolehmainen, M. Lassas, K. Niinimäki, and S. Siltanen. Sparsity-promoting Bayesian inversion. Inverse Problems, 28(2):025005, 2012.

18. Cristina Lavini and Joost J.C. Verhoeff. Reproducibility of the gadolinium concentration measurements and of the fitting parameters of the vascular input function in the superior sagittal sinus in a patient population. Magnetic Resonance Imaging, 28(10):1420-1430, 2010.

19. M. Lustig, D. Donoho, and J. M. Pauly. Sparse mri: The application of compressed sensing for rapid mr imaging. Magnetic Resonance in Medicine, 58:118-195, 2007.

20. Laura Martincich, Filippo Montemurro, Giovanni De Rosa, Vincenzo Marra, Riccardo Ponzone, Stefano Cirillo, Marco Gatti, Nicoletta Biglia, Ivana Sarotto, Piero Sismondi, Daniele Regge, and Massimo Aglietta. Monitoring response to primary chemotherapy in breast cancer using dynamic contrast-enhanced magnetic resonance imaging. Breast Cancer Research and Treatment, 83(1):67-76, 2004.

21. Zamir Merali, Kun Huang, David Mikulis, Frank Silver, and Andrea Kassner. Evolution of blood-brain-barrier permeability after acute ischemic stroke. PLoS One, 12(2):1-11, 2017.

22. K. Niinimäki, M. Lassas, K. Hämäläinen, A. Kallonen, V. Kolehmainen, E. Niemi, and S. Siltanen. Multi-resolution parameter choice method for total variation regularised tomography. submitted, http://arxiv.org/abs/1407.2386, 2015.

23. Martin Pickles, Martin Lowry, David Manton, Peter Gibbs, and Lindsay Turnbull. Role of dynamic contrast enhanced MRI in monitoring early response of locally advanced breast cancer to neoadjuvant chemotherapy. Breast Cancer Research and Treatment, 91(1):1-10, 2005.

24. Francesca Piludu, Simona Marzi, Andrea Pace, Veronica Villani, Alessandra Fabi, Carmine Carapella, Irene Terrenato, Anna Antenucci, and Antonello Vidiri. Early biomarkers from dynamic contrast-enhanced magnetic resonance imaging to predict the response to antiangiogenic therapy in high-grade gliomas. Neuroradiology, 57(12):1269-1280, 2015. 
25. Ruediger E. Port, Michael V. Knopp, and Gunnar Brix. Dynamic contrastenhanced MRI using Gd-DTPA: Interindividual variability of the arterial input function and consequences for the assessment of kinetics in tumors. Magnetic Resonance in Medicine, 45(6):1030-1038, 2001.

26. L.I. Rudin, S. Osher, and E. Fatemi. Nonlinear total variation based noise removal algorithms. Physica D: Nonlinear Phenomena, 60(1-4):259-268, 1992.

27. Kusmia S., Eliav U., Navon G., and Guillot G. Dqf-mt mri of connective tissues : application to tendon and muscle. Magnetic Resonance Materials in Physics Biology and Medicine, 26:203214, 2013.

28. Paul S. Tofts, Gunnar Brix, David L. Buckley, Jeffrey L. Evelhoch, Elizabeth Henderson, Michael V. Knopp, Henrik B. W. Larsson, Ting Yim Lee, Nina A. Mayr, Geoffrey J. M. Parker, Ruediger E. Port, June Taylor, and Robert M. Weisskoff. Estimating kinetic parameters from dynamic contrastenhanced T1weighted MRI of a diffusable tracer: Standardized quantities and symbols. Journal of Magnetic Resonance, 10(3):223-232, 1999.

29. Pedro Antonio Valds-Hernndez, Akira Sumiyoshi, Hiroi Nonaka, Risa Haga, Eduardo AubertVsquez, Takeshi Ogawa, Yasser Iturria-Medina, Jorge J. Riera, and Ryuta Kawashima. An in vivo MRI template set for morphometry, tissue segmentation, and fMRI localization in rats. Frontiers in Neuroinformatics, 5:26, 2011.

30. Kersten Villringer, Borja E. Sanz Cuesta, Ann-Christin Ostwaldt, Ulrike Grittner, Peter Brunecker, Ahmed A. Khalil, Kristina Schindler, Ole Eisenbltter, Heinrich Audebert, and Jochen B. Fiebach. DCE-MRI bloodbrain barrier assessment in acute ischemic stroke. Neurology, 88(5):433-440, 2017.

31. Dong Wang, Lori R. Arlinghaus, Thomas E. Yankeelov, Xiaoping Yang, and David S. Smith. Quantitative evaluation of temporal regularizers in compressed sensing dynamic contrast enhanced MRI of the breast. International Journal of Biomedical Imaging, page 7835749, 2017.

32. Stefanie Winkelmann, Tobias Schaeffter, Thomas Koehler, Holger Eggers, and Olaf Doessel. An optimal radial profile order based on the golden ratio for time-resolved MRI. IEEE Transactions on Medical Imaging, 26(1):68-76, 2007.

33. X. Zong, J. Lee, A.J. Poplawsky, Kim S.-G., and C.Y. Jong. Compressed sensinf fmri using gradient-recalled echo and epi sequences. NeuroImage, 92:312-321, 2014. 


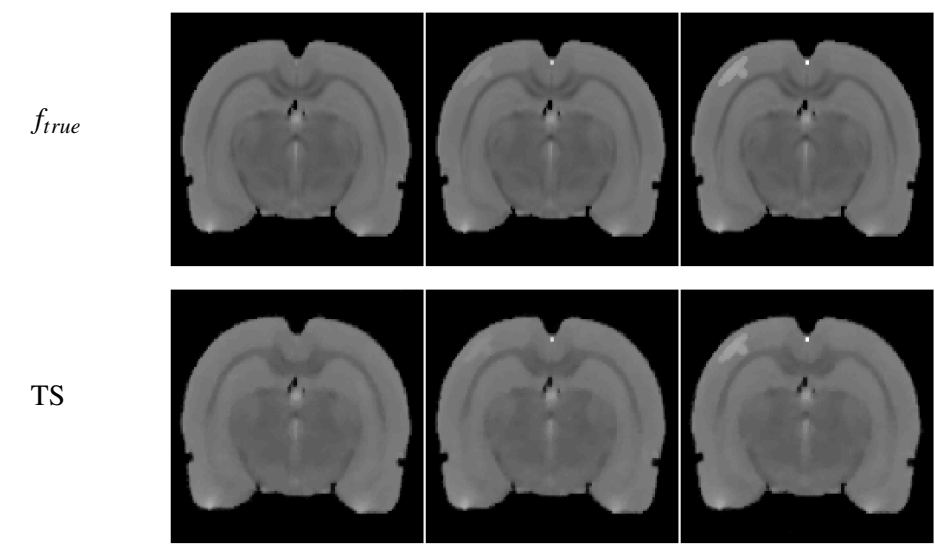

TV

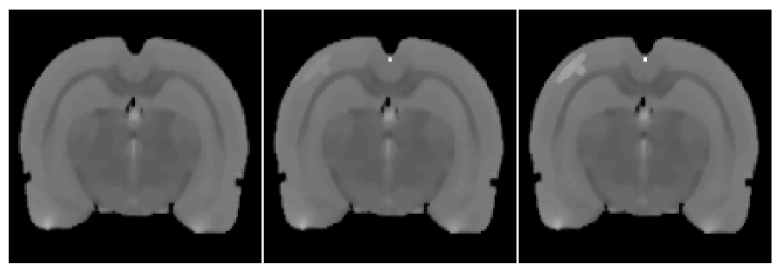

TGV

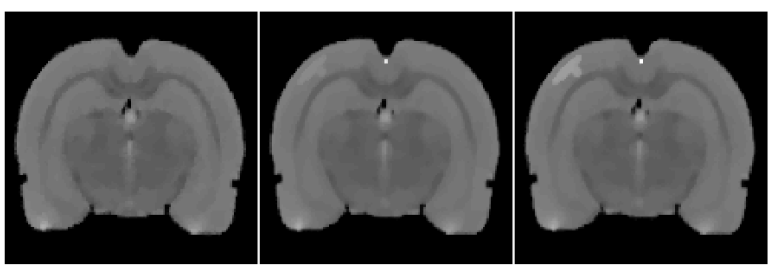

Regrid
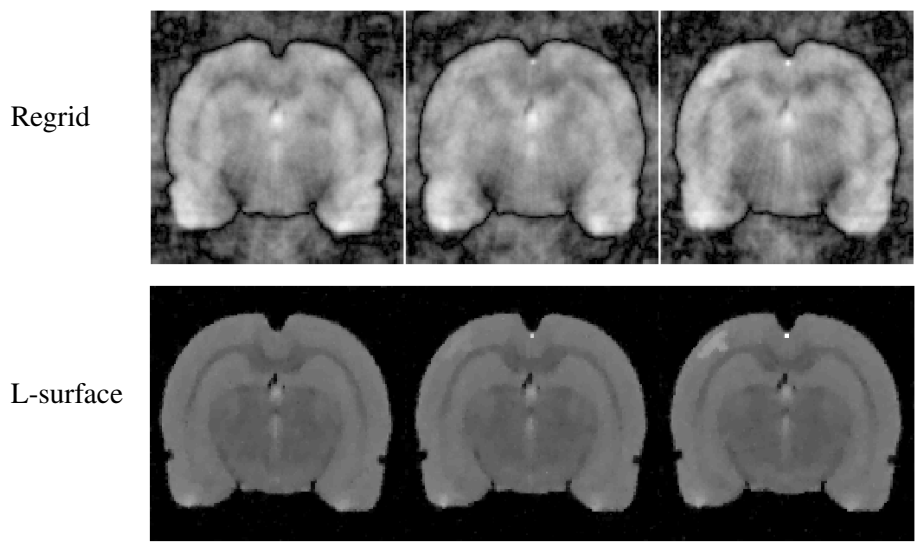

Fig. 4 Reconstructions with different temporal regularizations at different time frames. From top to bottom: true phantom, TS, TV, TGV, regrid and L-surface. From left to right time frames: before, during and after CA administration. 

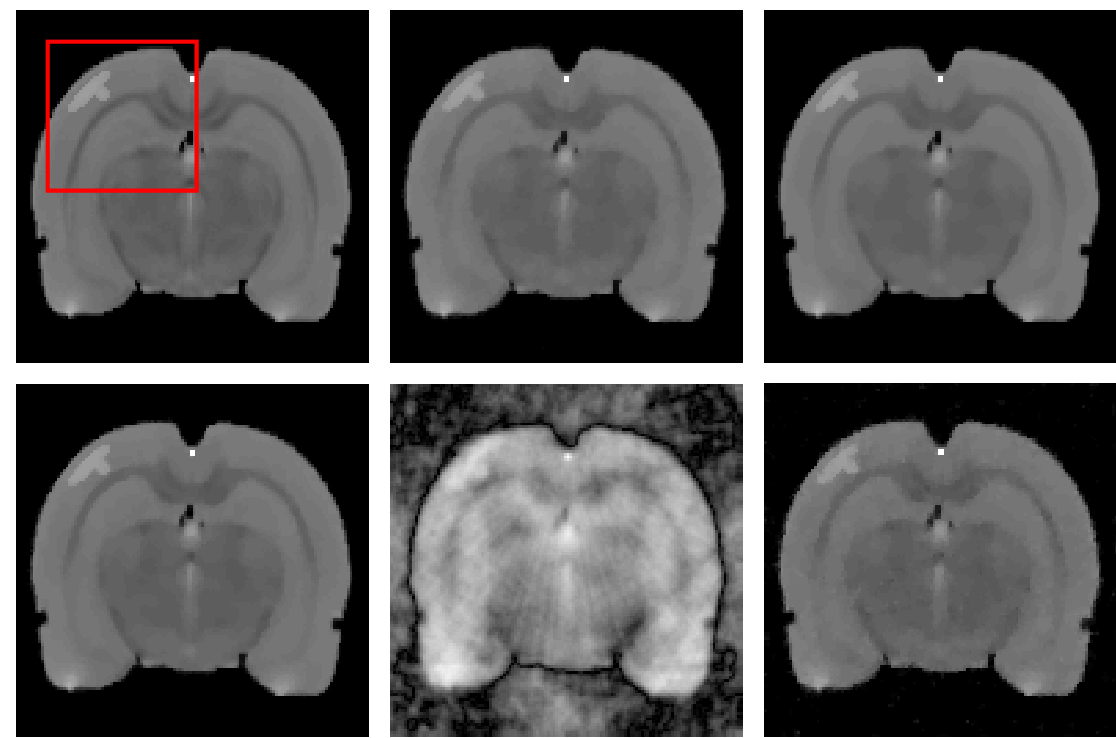

Fig. 5 Reconstructions with different temporal regularizations after CA administration. Top row: true phantom (left), TS reconstruction (middle) and TV reconstruction (right). Bottom row: TGV reconstruction (left), regrid-reconstruction (middle) and L-surface reconstruction (right). The area highlighted in red is presented as closeups in figure 6
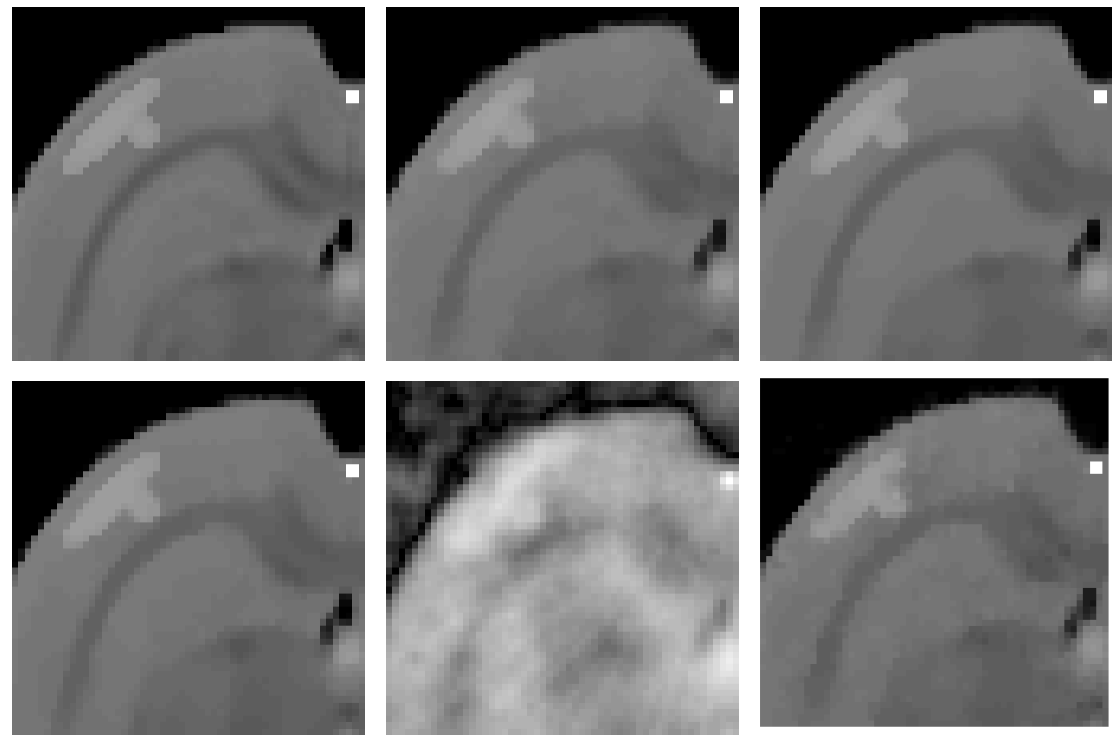

Fig. 6 Closeups of reconstructions with different temporal regularization methods after CA administration. Top row: true phantom (left), TS reconstruction (middle) and TV reconstruction (right). Bottom row: TGV reconstruction (left), regrid-reconstruction (middle) and L-surface reconstruction (right). 

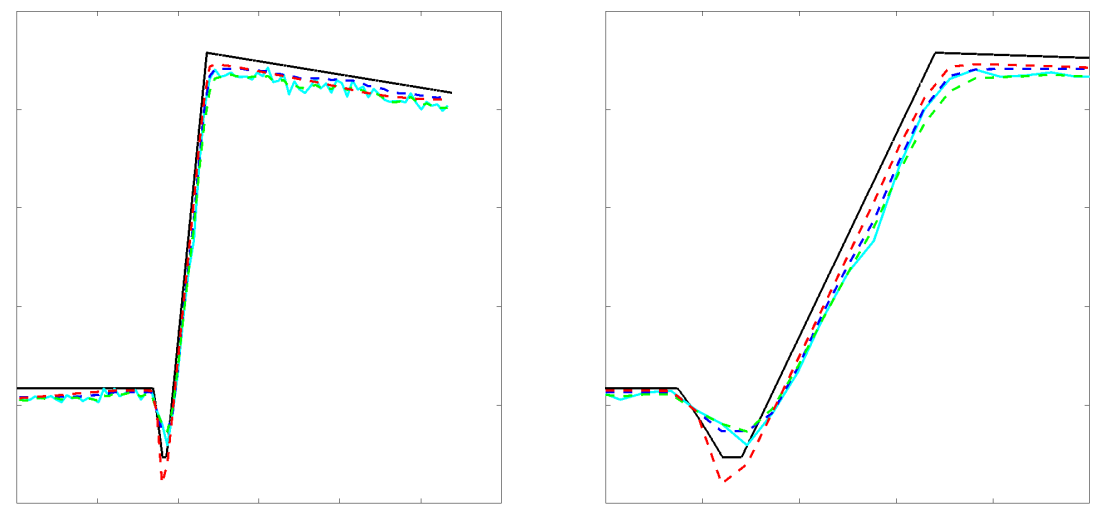

Fig. 7 Reconstructions of vascular region $\left(\Omega_{\text {roil }}\right)$ with the different methods at their optimal parameters according to the joint RMSE. Black line: true signal, blue line: TV reconstruction, green line: TS reconstruction, red line: TGV reconstruction and light blue line: L-surface reconstruction. Left: temporal evolution during all time frames. Right: closeup image during CA administration.
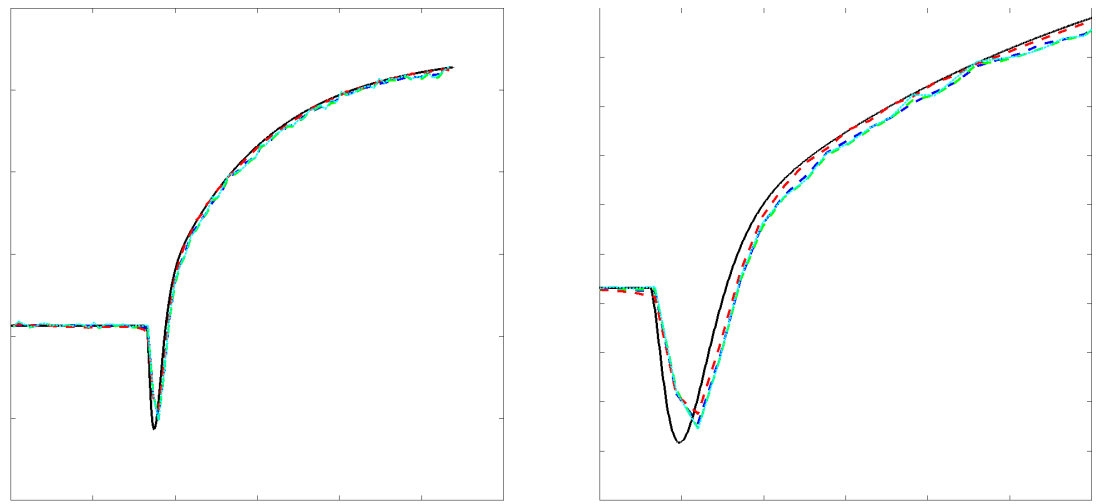

Fig. 8 Reconstructions of tumor region $\left(\Omega_{\text {roi }}\right)$ with the different methods at their optimal parameters according to the joint RMSE. Black line: true signal, blue line: TV reconstruction, green line: TS reconstruction, red line: TGV reconstruction and light blue line: L-surface reconstruction. Left: temporal evolution during all time frames. Right: closeup image during CA administration. 